\title{
Outcome after a new porous tantalum rod implantation for treatment of early-stage femoral head osteonecrosis
}

\author{
Wei Huang ${ }^{1}$, Xuan Gong ${ }^{2}$, Steve Sandiford ${ }^{1,3}$, Xiaoqiang $\mathrm{He}^{1,4}$, Feilong $\mathrm{Li}^{1,5}$, Yuwan $\mathrm{Li}^{1}$, Ziming Liu ${ }^{1}$, \\ Leilei Qin ${ }^{1}$, Jianye Yang ${ }^{1}$, Sizheng Zhu ${ }^{1}$, Jiawei Wang ${ }^{1}$, Xiaolin $\mathrm{Tu}^{6}$, Lei Ye ${ }^{7}$, Ning $\mathrm{Hu}^{1}$ \\ ${ }^{1}$ Department of Orthopedics, The First Affiliated Hospital of Chongqing Medical University, Chongqing 400016, China; ${ }^{2}$ Department of \\ Outpatient, Chongqing General Hospital, Chongqing, 400013, China; ${ }^{3}$ Department of Orthopedics, Queen Elizabeth Hospital, Bridgetown, \\ Barbados; ${ }^{4}$ Department of Orthopedics, The Third Affiliated Hospital of Chongqing Medical University, Chongqing 400016, China; ${ }^{5}$ Department \\ of Orthopedics, Chongqing Dazu People's Hospital, Chongqing 402360, China; ${ }^{6}$ Laboratory of Skeletal Development and Regeneration, Institute of \\ Life Sciences, Chongqing Medical University, Chongqing 400016, China; ${ }^{7}$ Chongqing Runze Pharmaceutical Co. Ltd., Chongqing 401120, China \\ Contributions: (I) Conception and design: N Hu, W Huang; (II) Administrative support: W Huang, X Tu, L Ye; (III) Provision of study materials or \\ patients: X He, Y Li, Z Liu; (IV) Collection and assembly of data: F Li, L Qin, J Yang, S Zhu, J Wang; (V) Data analysis and interpretation: X Gong, \\ S Sandiford; (VI) Manuscript writing: All authors; (VII) Final approval of manuscript: All authors. \\ Correspondence to: Prof. Ning Hu. Department of Orthopedics, The First Affiliated Hospital of Chongqing Medical University, Chongqing 400016, \\ China. Email: 1276321387@qq.com.
}

Background: Tantalum rods have been used in osteonecrosis of the femoral head (ONFH) for several years, while Zimmer trabecular metal implants have been proposed as the best choice. The aim of this study was to evaluate the effect of a new porous tantalum rod on the treatment of early ONFH.

Methods: From July 2014 to December 2015, 19 patients (21 hips) were treated with Runze tantalum rod, and 20 patients (20 hips) received Zimmer tantalum prosthesis. All patients were followed up for at least 3 years.

Results: There was no significant difference in demographic characteristics and the Harris Hip Score (HHS) improvement between the two groups. Kaplan-Meier analysis did not show any statistically significant difference in survival rates. One case in the Runze group had persistent pain and required conversion to total hip arthroplasty (THA) 8 months post-surgery. Histological evaluations revealed the presence of abundant new bone ingrowth into pores of the tantalum. The osteonecrosis observed in other patients was almost unchanged. At final follow-up, progressive collapse of the femoral head or the apparent joint space narrowing had not occurred.

Conclusions: Compared with the traditional implants, implantation of the Chinese tantalum rod in the treatment of Association Research Circulation Osseous (ARCO) stages I, and II ONFH demonstrated highly encouraging clinical results.

Keywords: Porous tantalum rod; osteonecrosis; femoral head; early stage; total hip arthroplasty (THA)

Submitted Apr 11, 2019. Accepted for publication Aug 08, 2019.

doi: $10.21037 /$ atm.2019.08.86

View this article at: http://dx.doi.org/10.21037/atm.2019.08.86

\section{Introduction}

Osteonecrosis of the femoral head (ONFH) is characterized by bone ischemia and microarchitectural deterioration. It is a multi-factorial process that leads to the collapse of the femoral head during the late stage. Intraosseous hypertension, thrombotic intravascular occlusion, and extravascular compression by progressive accumulation of marrow fat stores are the commonly accepted explanatory theories (1). In 1990, the Association Research Circulation Osseous (ARCO) held a meeting in Italy to advise on the staging and terminology of femoral head necrosis, which was revised in $1992(2,3)$. The recommendations were 
that ARCO I (positive bone scan or magnetic resonance imaging (MRI) positive or both positive, depending on the location of the femoral head involved, with the lesion subdivided into medial, central, and lateral parts) and ARCO II should be defined as early-stage of ONFH $(2,3)$. The primary goals of early-stage surgical treatment are to preserve the femoral head, relieve hip pain, and delay the progression of the disease. The joint-preserving procedures include restricted weight-bearing, electrical stimulation, core decompression (CD), rotational osteotomy, and nonvascularized and vascularized fibular grafting $(4,5)$. The choice of procedure is dependent upon the stage and the extent of necrosis; however, there is no consensus as to which category of patient is best served by which procedure. $\mathrm{CD}$ has been verified as a commonly effective surgery best used appropriately in small-sized and pre-collapse stages of osteonecrosis. However, isolated CD cannot delay the progression because of the lack of subchondral support $(6,7)$. Free vascularized fibula grafting can provide structural augmentation to the subchondral plate (8), but some of the difficulties in utilizing this option are its high degree of technical difficulty, longer operative time, high donor site morbidity, and prolonged rehabilitation $(4,9)$.

Porous tantalum rod implantation has been widely applied to patients with early-stage ONFH, in whom neither collapse of the femoral head nor any crescent line is noted (7). Most proponents of this method emphasize its advantages of $\mathrm{CD}$, enhanced bone in-growth due to the porous surface of the rod, and prevention of further collapse of the femoral head (10). Tantalum rod implantation has been widely applied to early $\mathrm{ONFH}$ in the clinic. However, the widespread use of a tantalum rod which at present is solely available from Zimmer Trabecular Metal Technology (Allendale, NJ, USA) has been considered expensive by other authors (11-13). It was further suggested that this ought to use only as a stop-gap or as a technique to "buy time" $(11,14)$. For the widespread application in the Chinese population, then, the development of a new tantalum implant would need to take the characteristics of the national economy into consideration. The presently accepted method of producing porous tantalum is to obtain a low-density precursor of a vitreous carbon skeleton with an interconnecting array of pores; pure tantalum is then deposited into and about the carbon skeleton to create a porous metal construct (15).

Runze Pharmaceutical Corporation (Chongqing, China) has produced a new porous tantalum material, which is prepared by slip-casting powder through teeming technology. It has a high porosity of $65-80 \%$, a mean pore size of $450 \mu \mathrm{m}$, and is able to induce the growth of bone tissue with rich blood vessels inside its pores. It is better than the $60-75 \%$ porosity, and the $430 \mu \mathrm{m}$ mean pore size of the Zimmer tantalum rod, and the biomechanical performance characteristics are comparable to human bone (11). Studies on new tantalum rod implantation for $\mathrm{ONFH}$ are as of yet unavailable. A few in vitro and in vivo studies evaluating biocompatibility and osteogenic properties of Runze porous tantalum implant in rabbits have been reported, however (16). Despite this, there have been no published comparative human trials between the use of new tantalum rods and Zimmer prostheses in earlystage ONFH.

The purpose of this study was to evaluate the early results of Runze porous tantalum rod in the treatment of early-stage ONFH, by means of comparing its outcome with a similar product from Zimmer. The second objective was to determine if the Runze tantalum rod would have good biocompatibility with superior bony ingrowth.

\section{Methods}

\section{Patients and surgical technique}

This prospective randomized clinical trial was approved by the Institutional Review Board and Hospital Ethics Committee of Chongqing Medical University, on July 22nd, 2014 (approval no. 2014-01-3). The period of study was from July 2014 to December 2015. The Chinese Clinical Trial Registry (a non-profit organization, established according to both the WHO International Clinical Trials Register Platform Standard and Ottawa Group Standard) granted full approval of the study protocol, recruitment materials and consent form (http://www.chictr. org.cn; Registration No. ChiCTR1800015182; date of supplementary registration; March 12th, 2018). All methods were carried out in accordance with the approved ethical guidelines. After the study had been completely described to the participants, they all signed written informed consent forms.

The main inclusion criteria for participants were as follows: (I) age between 18 and 60 years; (II) $\mathrm{BMI}<40$; (III) ARCO I, IIA, or IIB; (IV) non-traumatic etiologies; (V) no history of mental illness, drug abuse, systemic disease, infectious diseases, or immune system disorders (12-14). Exclusion criteria were as follows: (I) being actively treated with corticosteroids; (II) a history of previous surgery 


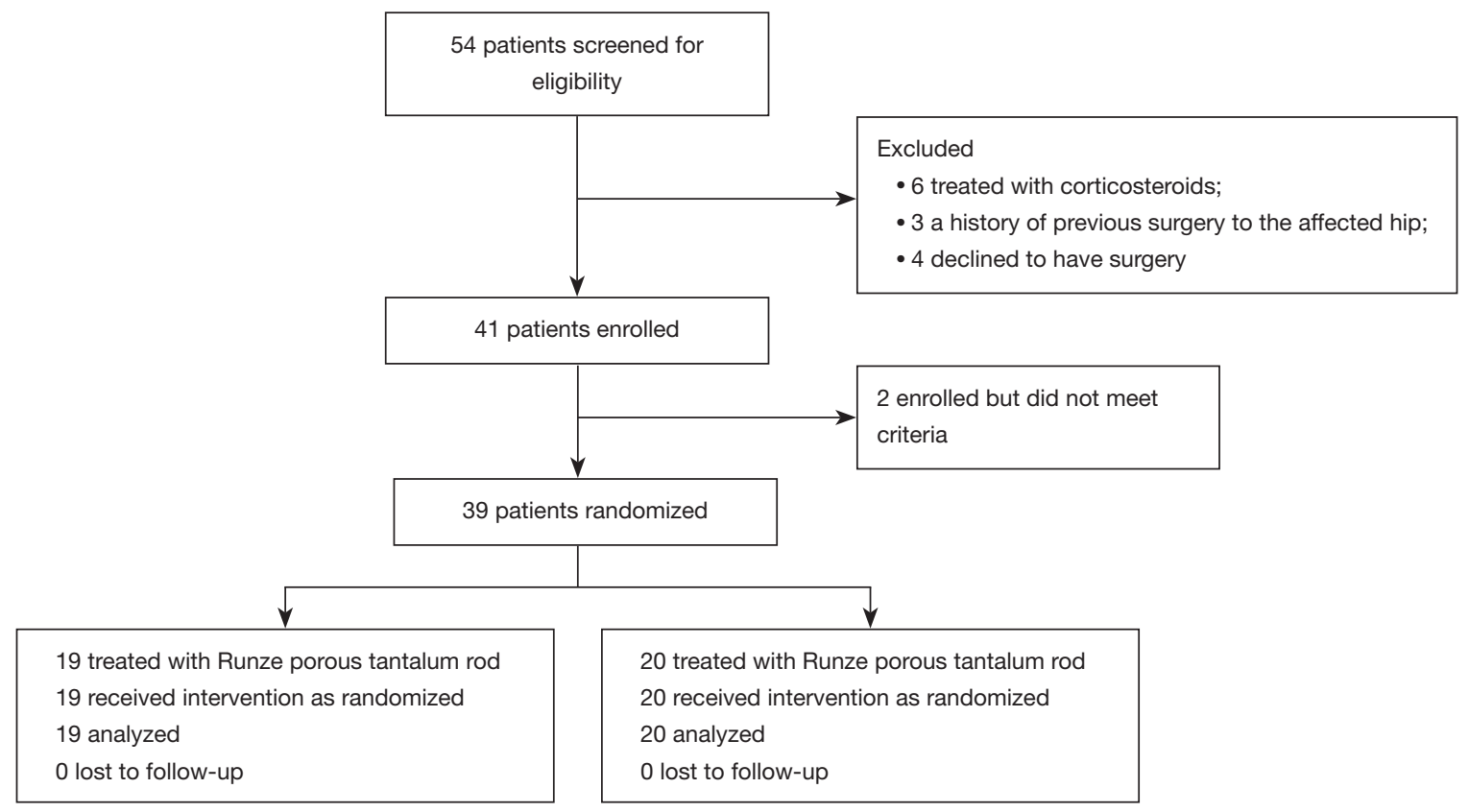

Figure 1 Study CONSORT diagram.

to the affected hip; (III) declining surgery at the time of clinical presentation (15). A flow diagram with the number of patients enrolled, excluded, and lost to follow-up is available in Figure 1. Randomization was done by nurses who drew a sealed, numbered envelope containing the treatment method for each patient prior to surgery. Among the patients, 19 (21 hips) were treated with Runze porous tantalum rod, and 20 (20 hips) received Zimmer tantalum prosthesis, in keeping in line with the same implant surgical technique description $(12,15)$.

All operations were performed by the same senior surgeon. The patients were placed in the supine position. Fluoroscopy was correlated with preoperative magnetic resonance imaging (MRI) and was used to detect the necrotic lesion center. A minimally invasive approach $(3 \mathrm{~cm})$ was made along the lateral thigh. The fascia lata and the vastus lateralis muscles were split longitudinally. A guide pin was inserted into the center of the necrotic lesion under biplanar fluoroscopic guidance, followed by core reamers, $8-10 \mathrm{~mm}$ in diameter, step-by-step over the guide pin to create a bone channel down to a depth of approximately $5 \mathrm{~mm}$ under the articular cartilage surface. Necrotic bone was removed thoroughly from the bone channel, which was the $\mathrm{CD}$ process. The autologous bone chips were placed in the excavated region of the femoral head. A porous tantalum rod of suitable length was threaded into the proper position after measuring and tapping. Finally, the incision was closed in layers without any drainage. Postoperative care consisted of prophylactic antibiotics, anticoagulation therapy, and protected weight-bearing for 3 months.

\section{The characteristics of Runze porous tantalum rod}

The materials were registered and tested by the Tianjin Medical Device Inspection Institute of China Food and Drug Administration (CFDA) (2010-GJ-600). The tantalum rod (TaBw01, Runze; patent no.: ZL 201110099357.6) is made entirely of pure porous tantalum, with a fully interconnected porosity of $65-80 \%$, a mean pore size of $450 \mu \mathrm{m}$, elastic modulus ( $3 \mathrm{GPa}$ ), a compressive strength of $40 \mathrm{Mpa}$, a flexure strength of $35 \mathrm{Mpa}$, physiological loadcarrying capability, and high frictional stability against bone $(11,15)$ (Figure 2). It has a $10-\mathrm{mm}$-diameter cylindrical shape with length options ranging from 70 to $130 \mathrm{~mm}$ (in 5 -mm increments). A thread section $(14 \mathrm{~mm}$ in diameter and $25 \mathrm{~mm}$ in length) is designed to engage the lateral cortex of the femur while a hemispherical tip supports the subchondral plate (Figure 2C).

\section{Clinical evaluation}

Post-operatively, follow-up examinations were scheduled 

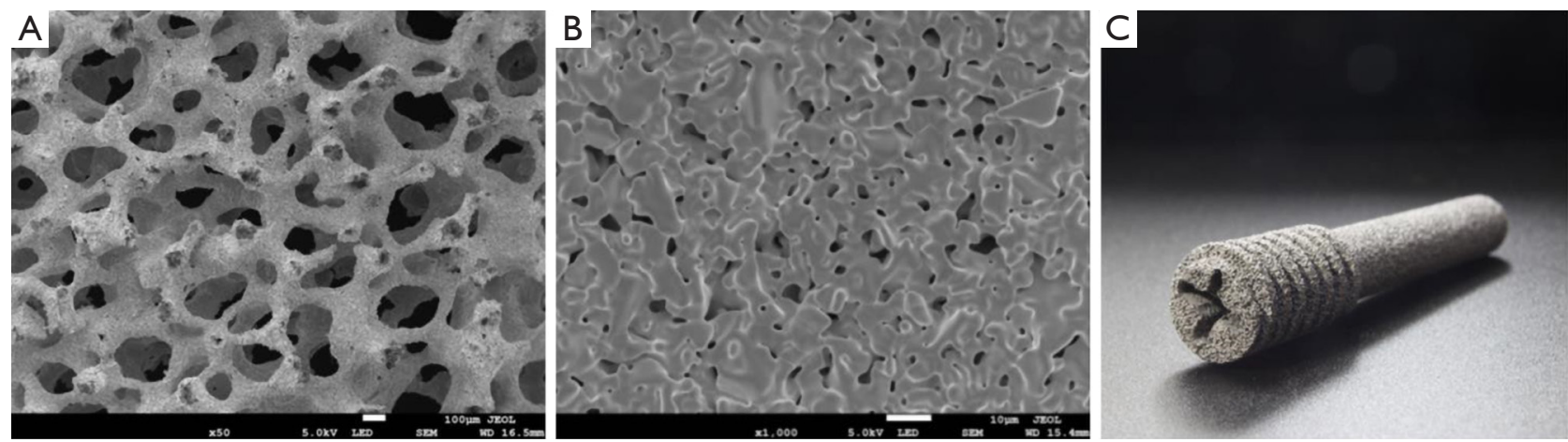

Figure 2 Surface characteristics of the tantalum rod (TaBw01, Runze). (A,B) Scanning electron micrograph revealed interconnected pore distribution with a pore diameter size of 400-600 $\mu \mathrm{m}$ and micropore structure of trabecular pillars; (C) photograph of the porous tantalum implant.

at 3 weeks, 6 weeks, 3 months, 6 months, and 1 year, and yearly thereafter. To assess clinical results, we compared the pre-operative and the post-operative Harris Hip Scores (HHS). Surgical time and blood loss were recorded as a supplement. The initial stage and extent of ONFH were assessed by plain radiographs or MRI according to the ARCO classification (10). We compared plain radiographs and MRI before and after the operation to document the progression of necrosis. MR images were used to evaluate bone marrow edema and changes in the size of the lesion. Throughout the study, a clinical evaluation was performed by a single observer who was not the surgeon and correlated with the preoperative MRI.

Two independent observers who were blinded to all clinical information performed the radiographic evaluation. Failure was defined as progression to femoral head collapse and was assessed by radiographic imaging or consistent pain following operation (4).

\section{Conversion to total hip arthroplasty (THA)}

The indications for conversion to THA included persistent hip pain, the radiological collapse of the femoral head, destructive arthritis, and intra-articular penetration of the tantalum rod. We osteotomized the femoral neck in the standard way using an oscillating saw. A $15-\mathrm{mm}$ trephine was used to extract the remaining portion. The lateral cortical hole was packed with a bone graft from the femoral head. The procedure was completed as an uncomplicated primary hip arthroplasty using a cementless prosthesis with ceramic on a highly cross-linked polyethylene-bearing surface.

\section{Porous tantalum bone-hard tissue slicing and Van Gieson (VG) staining}

The bone tissue in the upper femur was removed along with the porous tantalum rod, and then washed, trimmed, and fixed in $10 \%$ formaldehyde (11). The specimen was dehydrated using increasing concentrations of alcohol, then embedded in polymethylmethacrylate (Polysciences and Sigma-Aldrich). A diamond wafering saw (Isomer 1000, Buehler, Lake Bluff, IL, USA) was used to section the specimen along the direction parallel to the longitudinal axis of the rod. The plane was polished, and $90-\mu \mathrm{m}$ slices were prepared, which were ground down to $20 \mu \mathrm{m}$, dried and exposed to glycol ether ester to remove any plastic (11). Image acquisitions and assessments of bone callus growth were performed under an optical microscope after VG staining (16).

\section{Statistical analysis}

The Welch's $t$-test analysis was used to compare continuous variables such as demographic data, surgical time, blood loss, HHS between the two groups. Statistical differences in survival rates were calculated using log-rank Chisquare analysis of Kaplan-Meier survival curves, with the endpoint as required for THA. $\mathrm{P}$ values less than 0.05 were considered significant.

\section{Results}

\section{Surface characteristics of Runze porous tantalum rod}

Scanning electron microscopy (SEM) revealed that the 
Table 1 Demographic characteristics of the patients

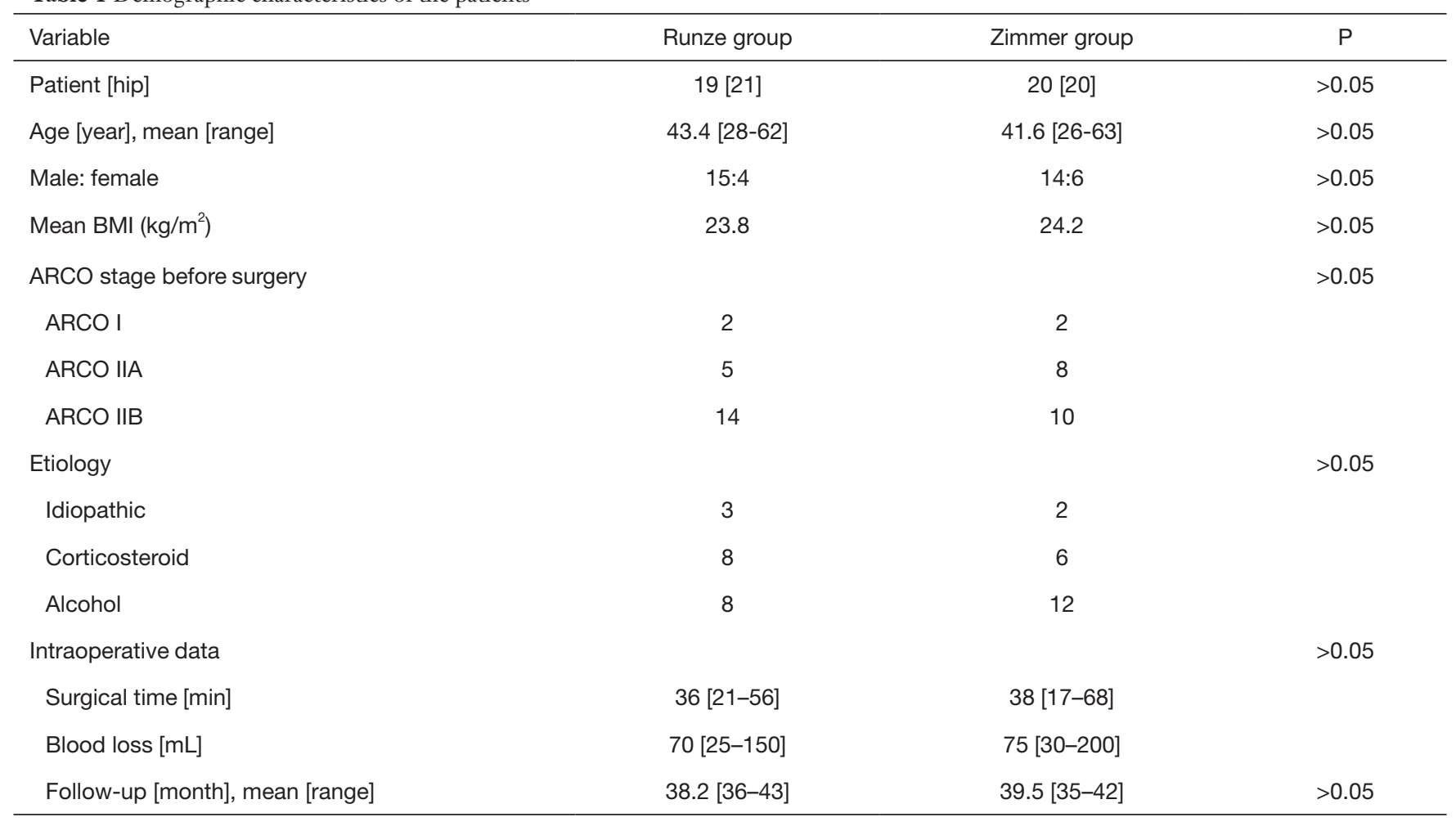

ARCO, Association Research Circulation Osseous.

porous tantalum was characterized by 65 to $80 \%$ porosity, a cellular pore size of 400-600 $\mu \mathrm{m}$, and 3D interconnecting pores (Figure 2A,B). Pore distribution was uniform, and trabecular pillars had a microporous structure. This unique structure provides strong local mechanical support while allowing bone ingrowth into the interconnecting pores.

\section{Demographic data}

According to the ARCO classification, there were 2 hips with stage I ONFH, 5 hips with stage IIA, and 14 hips with stage IIB in the Runze group. Mean follow-up was 38.2 months (range, 36-43 months). In the Zimmer group, 2 hips were classified as stage I, 8 hips were stage IIA, and 10 hips were stage IIB. Mean follow-up was 39.5 months (range, 35-42 months). No patient was lost to follow-up. There were no significant differences in age, sex, BMI, ARCO staging, and etiology between the two study populations. Mean operation time was $36 \mathrm{~min}$ (range, 21-56 min) in the Runze group and $38 \mathrm{~min}$ (range, 17-68 $\mathrm{min}$ ) in the Zimmer group. As recorded, the average blood losses of the two groups, respectively were $70 \mathrm{~mL}$ (range, 25-150 mL) and $75 \mathrm{~mL}$ (range, 30-200 mL) (Table 1).

\section{Changes in HHSs after treatment}

The average HHS was 62 preoperatively, 80 at 3 months, 82 at 6 months, 87 at 12 months, 90 at 18 months, 92 at 24 months, and 90 at 30 and 36 months in the Runze group (for the surviving hips) (Figure 3). One case was classified as a poor outcome and converted to a THA because of surgical error (Figure 4). The mean HHS within the Zimmer group increased postoperatively during follow-up from 64 to 92 (Figure 3). At a mean postoperative follow-up of 36 months, HHS significantly increased $(\mathrm{P}<0.001)$, with a higher score indicating improvement, compared with before surgery in both patient groups. There was no significant difference in the magnitude of HHS improvement between the two groups.

\section{Radiographic assessment}

Conventional radiograph and MRI confirmed the distal portion was inserted into the center of the area correctly, 


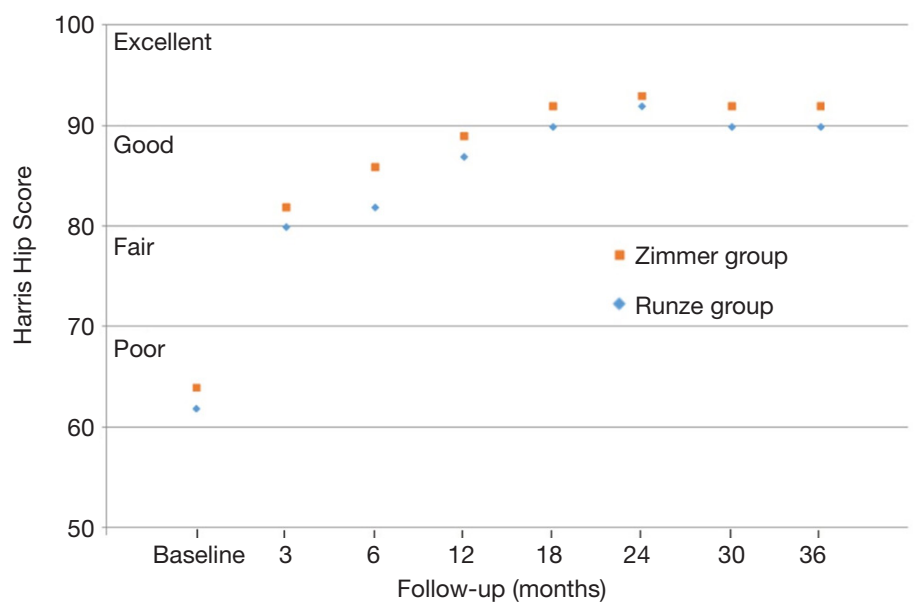

Figure 3 Harris Hip Score. Temporal trends of the Harris Hip Score in each treatment group over the entire follow-up period. Harris hip scores were significantly increased $(\mathrm{P}<0.0001)$ when compared to pre-surgery scores in both patient groups. The magnitude of increase in the Runze group was not significantly different from that in the Zimmer group.
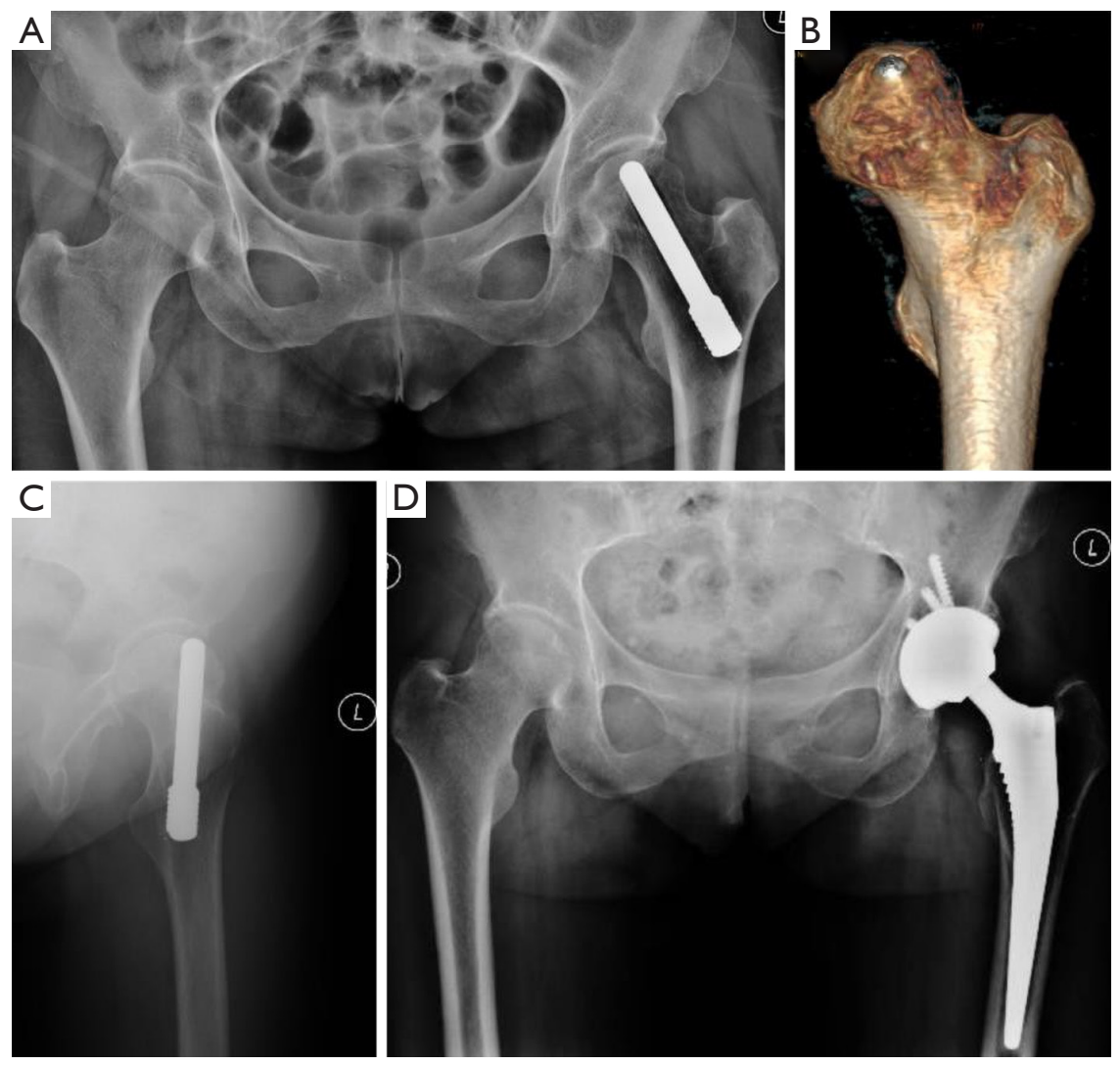

Figure 4 The case of conversion to THA. A 49-year-old female patient with left idiopathic necrosis in ARCO stage IIB. (A,B) Postoperative pelvic plain film and CT showed perforation of the articular surface and mistaken length of the tantalum rod; (C) the lateral view of the left hip was performed at the 8th month after implantation; (D) the Runze porous tantalum rod was replaced by THA in the revision operation at the 8th month of follow-up. High-density tantalum metal residue was noticed in the bone tunnel where the tantalum rod was inserted. THA, total hip arthroplasty; ARCO, Association Research Circulation Osseous. 

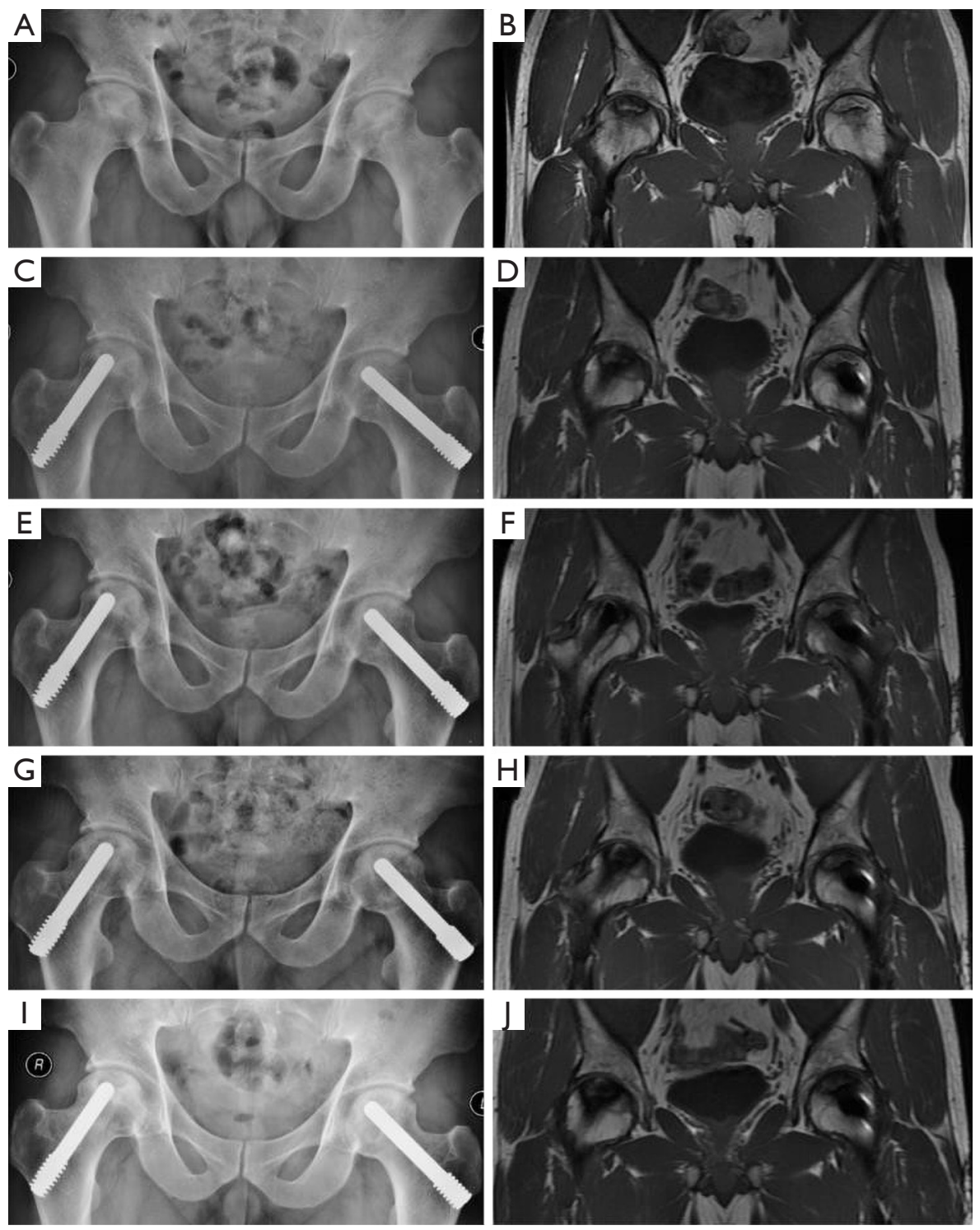

Figure 5 Representative case. Preoperative and three-year follow-up radiographs from a representative case of a 30-year-old male patient with steroid-associated bilateral necrosis in ARCO stage IIB treated with Runze porous tantalum implant technology. The X-rays and MRI has shown that they were taken before surgery (A,B), 6 months after surgery (C,D), 12 months after surgery (E,F), 24 months after surgery $(\mathrm{G}, \mathrm{H})$, and 36 months after surgery $(\mathrm{I}, \mathrm{J})$. Comparison of the postoperative and three-year follow-up radiographs demonstrate no change in implant position, no abnormal bone density, or radiolucent lines, and no apparent progression of osteonecrosis. ARCO, Association Research Circulation Osseous.

even in progressed necrosis of femoral heads. Post-operative radiology revealed the proper position of the implanted tantalum rod in all surviving hips (Figure 5). The progress of osteonecrosis in the patients who did not need a THA remained almost unchanged. At last follow-up, progressive collapse of the femoral head and/or the apparent joint space narrowing had not occurred. There were no significant differences in the radiological outcome between the two groups.

\section{Complications, survivorship, and conversion to THA}

In the Runze group, 1 case had persistent pain and unsatisfactory outcome and was converted to THA 


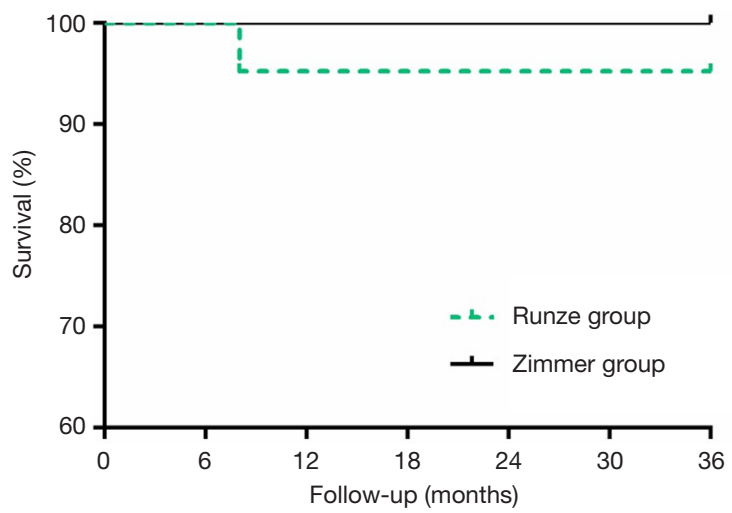

Figure 6 Kaplan-Meier survivorship curve. Survivorship curves for the hips in each treatment group, with the conversion to total hip replacement as the endpoint. The survival rate was $95.2 \%$ for the Runze group and $100 \%$ for the Zimmer group at 36 months postoperatively. Kaplan-Meier survivorship analysis showed no significant difference between the two groups (Chi square $=0.106$, $\mathrm{P}=0.764)$.
8 months post-surgery (Figure 4). The failure was secondary to perforation of the articular surface and mistaken length of the tantalum rod (Figure 4B,C). For the patient who underwent THA, high-density metal particle residuals were seen on the radiograph in the bone channel where the rod was inserted (Figure 4D). No patient was lost to followup, and no other complications, such as subtrochanteric fracture, infection, and deep vascular thrombosis occurred during the follow-up period. All other patients who had a hip survival time longer than 3 years were still doing well with good functional scores (Figure 3) and unchanged radiological appearance. Kaplan-Meier analysis at 36 months revealed similar cumulative survival rates for the Runze implant and Zimmer implant (Figure 6).

\section{Morphological analyses and VG staining}

No collapse of the femoral head and no gap between the host bone and the implant were visible by the naked eye (Figure $7 A, B, C)$. The implanted porous tantalum rod was
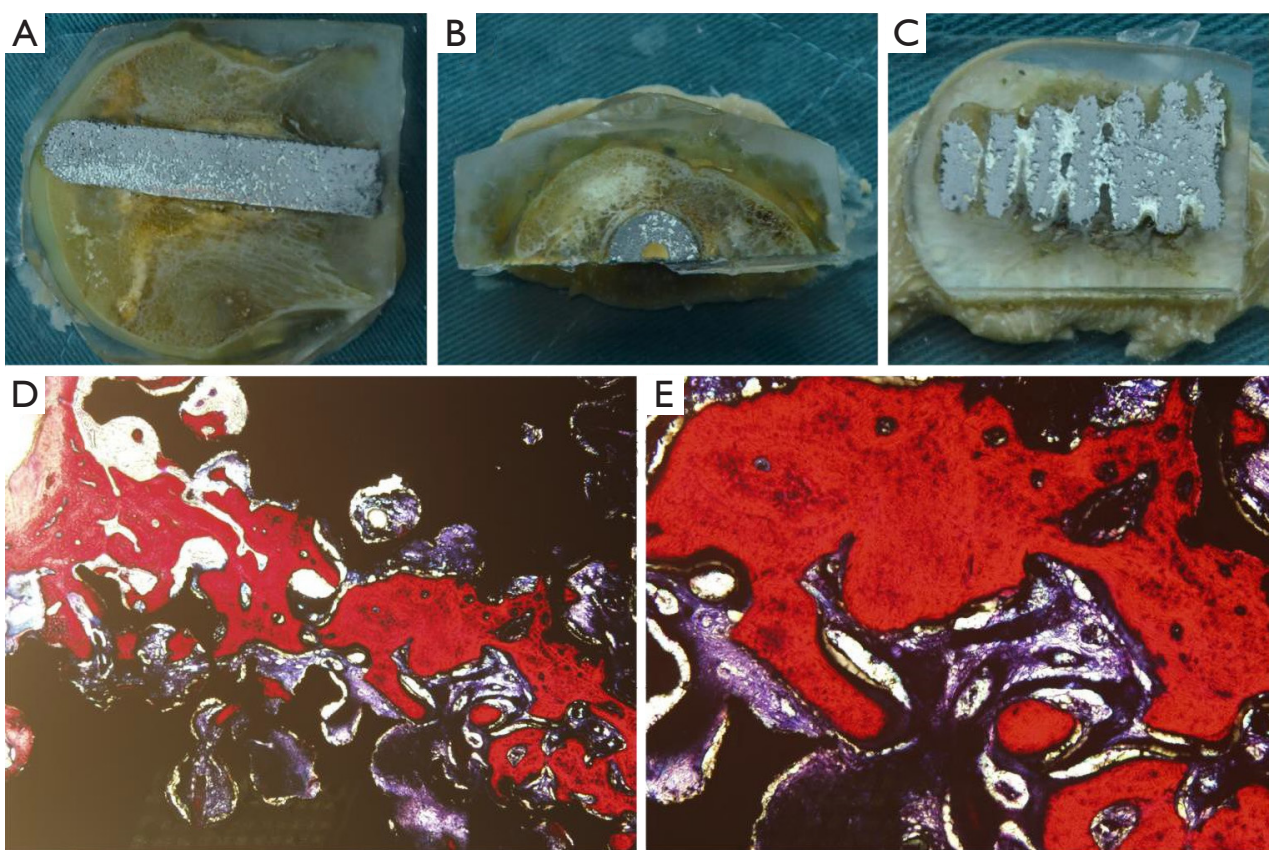

Figure 7 Histological observation of hard tissue slices prepared from porous tantalum implants at the 8th month after implantation (Van Gieson staining; magnification, $\times 40$, and $\times 100$ ). Photograph of the tantalum rod harvested in revision operation. Following dehydration, infiltration, embedding, and polymerization, a metal slicer was used to slice the material along the direction parallel to the longitudinal axis of the porous tantalum rod, fully exposing its plane. (A,B,C) Bone tissue was seen well integrated with the metal insertion. No collapse of the femoral head and no gap between the host bone and the implant were visible by the naked eye; (D,E) the red-dyed new bone grew along the pore walls. Abundant new bone ingrowth into the pores of the tantalum was observed, and some woven bones were interconnected. Fibrous septa and inflammatory response were not observed at the interface between the biomaterial and the bone. 
closely combined with the host bone, and loosening of implantation was not observed. The red-dyed new bone grew along the pore walls (Figure $7 D, E$ ). Abundant new bone ingrowth into the pores of the tantalum was observed, and some woven bones were interconnected.

\section{Discussion}

$\mathrm{ONFH}$ is a progressive and incapacitating disease, which typically affects the younger population and represents a remarkable challenge to orthopedic surgeons. Osteonecrosis often progresses to a stage of trabecular fracture, and subsequent subchondral bone collapse occurs, which leads to arthritis of the hip joint (4). Many joint-preserving surgeries have been used, such as $\mathrm{CD}$ and nonvascularized or vascularized bone grafting, with some successful results $(9,17)$, but, unfortunately, no definite solution exists $(18)$.

The possible benefits of a porous tantalum rod implantation were thought to include reduction of the intraosseous pressure, reperfusion with the possibility of regeneration, along with structural support, low donorsite morbidity, and minimal invasion $(4,7,12,14)$. This method was first proposed by Brown et al. in 1993 (6) who indicated that a porous tantalum rod was a reasonable mechanical substitute for a fibular graft. In a multicenter study, Tsao et al. reported a survival rate of $85 \%$ at 12 months, $79 \%$ at 24 months, and $73 \%$ at 48 months for all Steinberg stage II hips. The average HHS for all stage II hips was 63 preoperatively, 75 at six months, 78 at one and two years, and 86 at three years (5). Pakos et al. treated 49 patients (58 hips) with tantalum rod and autologous bone grafting and reported that the 5-year survival based on conversion to THA was $93.1 \%$, while the respective rate based on disease progression was $87.9 \%$ (19). Liu et al. treated 59 consecutive hips ( 45 patients) with porous tantalum implants and 2-mm composite bone filling material and reported that the overall survival rate was $72.49 \%$ at 60 months postoperatively, with 11 hips $(11 / 57,19.3 \%)$ being converted to THA (20). Reported failure rates following CD and tantalum rod implantation have ranged from $2 \%$ at 15 months to $56 \%$ at 18 months (21). Nadeau et al. reported a survival rate of $42.5 \%$ after 48 months and failure in 10 of 18 (55.6\%) tantalum implant procedures to treat Steinberg stages III and IV ONFH, with an average follow-up of 23.2 months (22). Although there was no statistical difference between Runze and Zimmer implants in our study, the survival rate was significantly higher than that reported in Tsao et al. (5), Liu et al. (20), and Nadeau et al. (22), and similar to that of Pakos et al. (19). Thus, Runze tantalum implants, with a $95 \%$ survival, appear to be a feasible option.

Previous studies have used Zimmer tantalum implants, which are relatively expensive and impose a heavy financial burden on patients. The cost has been considered when choosing the surgical procedure $(7,13)$. Currently, there are few reports on the treatment of ONFH with other new tantalum prostheses. Our analysis demonstrated that the new porous tantalum rod (TaBw01, Runze Pharmaceutical Corporation) was effective and well-suited for early ONFH patients (ARCO I, IIA, and IIB). Cytocompatibility studies confirmed vivid migration and proliferation of osteocyte in the pores of the material following implantation of Runze porous tantalum in the host bone, and Wang et al. found the existence of a solid combination of porous tantalum and bone, in addition to conduction in en vivo rabbit studies (11); however, this study period was only 3 months. Based on the longer clinical observation and histological evidence from the retrieved implant, it is clear that the porous surface of Runze tantalum rod supported bone tissue ingrowth or ongrowth and could be effective for supplementing the stability of the implant by biological fixation.

Zimmer porous tantalum, in its trabecular form, combines strong mechanical resistance with high biocompatibility and osteoconductive and osteoinductive properties (23). It has a high volumetric porosity, and its modular elasticity is similar to the subchondral bone, yet its strength and endurance limit is better than a natural bone graft $(17,24)$. The Chinese porous tantalum (TaBw01, Runze Pharmaceutical Corporation) also possesses a substantially high and interconnecting porosity $(65-80 \%)$ with an appropriate pore diameter size $(400-600 \mu \mathrm{m})(11,15)$. It allows the free flow of nutrients and uninhibited vascularization to the internal structure of the rod. Its three-dimensional geometric scaffold with honeycomb pores provides an ideal material-cell interface on which osteoblasts are able to adhere, proliferate, and develop. Wang et al. demonstrated that Chinese porous tantalum implants offered good biocompatibility (11). The new porous tantalum implant has similar flexural rigidity (35 MPa) to the human fibula. Its compressive strength (40 MPa) is between that of cortical and cancellous bone, and its elastic modulus (2.1 GPa) falls between that of cortical and subchondral bone, thereby providing mechanical support to the subchondral plate while limiting stress shielding.

Zhang et al. conducted a case series focused on the failure of tantalum used to treat ONFH with associated retrieval analysis. Admittedly, limited by small sample size 
(13 patients), the reported one-year survival rate was $64.29 \%$ and 4 patients converted to arthroplasty in a mean time of 305 days (15). Our success rate was conspicuously higher than Zhang et al.'s study. In Zhang et al.'s study, the postoperative load-bearing was not restricted, but we protected weight-bearing for 3 months after the operation. Thus, postoperative load bearing may be one of the reasons for this difference. We followed a group of 18 patients (19 hips) with Runze porous tantalum implants over an average of 36 months, with only 1 (5\%) patient failing. The reason for the failure was related to the implantation depth of the tantalum rod. Biplanar active fluoroscopy (BAF) and computer-aided surgery (CAS) are two techniques that can substantially improve the reliability of the core tract and implant placement (5). Using BAF and CAS may improve survival rate. Hard tissue slicing and staining of retrieved ONFH indicated the full ingrowth of bony tissue into the porous structure. Under higher magnification, it was observed that the new bone-ingrowth structure was distributed evenly on the inner surface of the pores. The clinical symptoms of the early stage patients improved according to the HHS and Western Ontario and McMaster Universities Osteoarthritis Index (WOMAC). Therefore, we advise that the tantalum rod should be indicated only in the early-stage of $\mathrm{ONFH}$, involving not more than $30 \%$ of the joint surface (23) and that Runze tantalum rod is considered for the first choice.

In addition to implants, there are many factors that affect prognosis. Size and location of necrotic involvement have been known to influence the outcomes of surgery. Additionally, the effectiveness of the implant may be severely limited by the etiology of osteonecrosis (25-27). The MRIbased volumetric method was used to quantify the size of the lesion on preoperative MRI scans $(28,29)$. However, many patients with early-stage prefer X-rays instead of MRI when they feel discomfort. Thus, patient selection has been an important prognostic factor for the progress of osteonecrosis. In addition, the appropriate position and proper length of tantalum implant are very important. The necrotic bone should be removed thoroughly from the bone channel, and cancellous bone chips should be placed in the excavated region of the femoral head (30). A disadvantage of this study was the relatively small number of patients and the different etiologies of the ONFH. The effect of particle residuals of the new tantalum on the long-term survivorship of THA requires further study. Retrieval analysis studies of porous tantalum tibial trays have been limited.

In conclusion, compared with traditional implants, the clinical and radiological outcomes of the Chinese porous tantalum rod are satisfactory, which makes it a favorable choice in the treatment of ARCO stages I or II ONFH.

\section{Acknowledgments}

Funding: this study was supported by the National Natural Science Foundation of China (NSFC) (Grant number $81672167,81371972,81572142$ and 81972069).

\section{Footnote}

Conflicts of Interest: The authors have no conflicts of interest to declare.

Ethical Statement: The authors are accountable for all aspects of the work in ensuring that questions related to the accuracy or integrity of any part of the work are appropriately investigated and resolved. This prospective randomized clinical trial was approved by the Institutional Review Board and Hospital Ethics Committee of Chongqing Medical University, on July 22nd, 2014 (Approval no. 2014-01-3).

\section{References}

1. Wang C, Huang W, Gong X, et al. Decreased proliferation and abnormal differentiation of human mesenchymal stromal cells in steroid-induced osteonecrosis of femoral head. SOJ Biochem 2014;1:8.

2. Gardeniers JWM. The ARCO perspective for reaching one uniform staging system of osteonecrosis. In: Schontens A, Arlet J, Gardeniers JWM, et al. editors. Bone circulation and vascularizatrion in normal and pathological conditions. Boston: Springer, 1993:375-80.

3. Mont MA, Hungerford DS. Non-tranmatie avascular necrosis of the femoral head. J Bone Joint Surg (Am) 1995;77:459.

4. Liu G, Wang J, Yang S, et al. Effect of a porous tantalum rod on early and intermediate stages of necrosis of the femoral head. Biomed Mater 2010;5:065003.

5. Tsao AK, Roberson JR, Christie MJ, et al. Biomechanical and clinical evaluations of a porous tantalum implant for the treatment of early-stage osteonecrosis. J Bone Joint Surg Am 2005;87 Suppl 2:22-7.

6. Brown TD, Pedersen DR, Baker KJ, et al. Mechanical consequences of core drilling and bone-grafting on osteonecrosis of the femoral head. J Bone Joint Surg Am 1993;75:1358-67.

7. Floerkemeier T, Thorey F, Daentzer D, et al. Clinical and 
radiological outcome of the treatment of osteonecrosis of the femoral head using the osteonecrosis intervention implant. Int Orthop 2011;35:489-95.

8. Cao L, Guo C, Chen J, et al. Free Vascularized Fibular Grafting Improves Vascularity Compared With Core Decompression in Femoral Head Osteonecrosis: A Randomized Clinical Trial. Clin Orthop Relat Res 2017;475:2230-40.

9. Zhao D, Zhang $\mathrm{Y}$, Wang $\mathrm{W}$, et al. Tantalum rod implantation and vascularized iliac grafting for osteonecrosis of the femoral head. Orthopedics 2013;36:789-95.

10. Lee GW, Park KS, Kim DY, et al. Results of THA after core decompression with tantalum rod for osteonecrosis of the femoral head. Clin Orthop Surg 2016;8:38-44.

11. Wang Q, Zhang H, Li Q, et al. Biocompatibility and osteogenic properties of porous tantalum. Exp Ther Med 2015;9:780-6.

12. Shuler MS, Rooks MD, Roberson JR. Porous tantalum implant in early osteonecrosis of the hip: preliminary report on operative, survival, and outcomes results. J Arthroplasty 2007;22:26-31.

13. Miao H, Ye D, Liang W, et al. Effect of osteonecrosis intervention rod versus core decompression using multiple small drill holes on early stages of necrosis of the femoral head: a prospective study on a series of 60 patients with a minimum 1-Year-Follow-Up. Open Orthop J 2015;9:179-84.

14. Liu Y, Yan L, Zhou S, et al. Tantalum rod implantation for femoral head osteonecrosis: survivorship analysis and determination of prognostic factors for total hip arthroplasty. Int Orthop 2016;40:1397-407.

15. Zhang X, Wang J, Xiao J, et al. Early failures of porous tantalum osteonecrosis implants: a case series with retrieval analysis. Int Orthop 2016;40:1827-34.

16. Ren B, Zhai Z, Guo K, et al. The application of porous tantalum cylinder to the repair of comminuted bone defects: a study of rabbit firearm injuries. Int J Clin Exp Med 2015;8:5055-64.

17. Zhang Y, Li L, Shi ZJ, et al. Porous tantalum rod implant is an effective and safe choice for early-stage femoral head necrosis: a meta-analysis of clinical trials. Eur J Orthop Surg Traumatol 2013;23:211-7.

18. Yu X, Zhang D, Chen X, et al. Effectiveness of various hip preservation treatments for non-traumatic osteonecrosis of the femoral head: A network meta-analysis of randomized controlled trials. J Orthop Sci 2018;23:356-64.

19. Pakos EE, Megas P, Paschos NK, et al. Modified porous tantalum rod technique for the treatment of femoral head osteonecrosis. World J Orthop 2015;6:829-37.
20. Liu Y, Su X, Zhou S, et al. A modified porous tantalum implant technique for osteonecrosis of the femoral head: survivorship analysis and prognostic factors for radiographic progression and conversion to total hip arthroplasty. Int J Clin Exp Med 2015;8:1918-30.

21. Olsen M, Lewis PM, Morrison Z, et al. Total hip arthroplasty following failure of core decompression and tantalum rod implantation. Bone Joint J 2016;98-B:1175-9.

22. Nadeau M, Séguin C, Theodoropoulos JS, et al. Short term clinical outcome of a porous tantalum implant for the treatment of advanced osteonecrosis of the femoral head. Mcgill J Med 2007;10:4-10.

23. Aldegheri R, Taglialavoro G, Berizzi A. The tantalum screw for treating femoral head necrosis: rationale and results. Strategies Trauma Limb Reconstr 2007;2:63-8.

24. Shimko DA, Shimko VF, Sander EA, et al. Effect of porosity on the fluid flow characteristics and mechanical properties of tantalum scaffolds J Biomed Mater Res B Appl Biomater 2005;73:315-24.

25. Yoo MC, Kim KI, Hahn CS, et al. Long-term followup of vascularized fibular grafting for femoral head necrosis. Clin Orthop Relat Res 2008;466:1133-40.

26. Floerkemeier T, Lutz A, Nackenhorst U, et al. Core decompression and osteonecrosis intervention rod in osteonecrosis of the femoral head: clinical outcome and finite element analysis. Int Orthop 2011;35:1461-6.

27. Escudier JC, Ollivier M, Donnez M, et al. Superimposition of maximal stress and necrosis areas at the top of the femoral head in hip aseptic osteonecrosis. Orthop Traumatol Surg Res 2018;104:353-8.

28. Varitimidis SE, Dimitroulias AP, Karachalios TS, et al. Outcome after tantalum rod implantation for treatment of femoral head osteonecrosis: 26 hips followed for an average of 3 years. Acta Orthop 2009;80:20-5.

29. Lee GW, Park KS, Kim DY, et al. Results of total hip arthroplasty after core decompression with tantalum rod for osteonecrosis of the femoral head. Clin Orthop Surg 2016;8:38-44.

30. Liu B, Sun W, Yue D, et al. Combined tantalum implant with bone grafting for the treatment of osteonecrosis of the femoral head. J Invest Surg 2013;26:158-62.

Cite this article as: Huang W, Gong X, Sandiford S, He X, Li F, Li Y, Liu Z, Qin L, Yang J, Zhu S, Wang J, Tu X, Ye L, Hu N. Outcome after a new porous tantalum rod implantation for treatment of early-stage femoral head osteonecrosis. Ann Transl Med 2019;7(18):441. doi: 10.21037/atm.2019.08.86 